\title{
State-dependent retention effects with xylazine (Rompun) in passive-avoidance conditioning
}

\author{
RUSSELL E. MORGAN and DAVID C. RICCIO \\ Kent State University, Kent, Ohio
}

\begin{abstract}
To determine whether xylazine (Rompun), an alpha-2 adrenergic receptor agonist with sedative/ analgesic properties, influences memory processes, rats were trained in a passive-avoidance task either before or after receiving the drug. Experiment 1 found that subjects who had received a 3-cc/kg Rompun injection (IP) $60 \mathrm{sec}$ before training showed impaired retention when tested $24 \mathrm{~h}$ later in a mismatched-drug condition. This impairment, however, was reversed in an identically trained group that received a second injection of the drug $60 \mathrm{sec}$ before testing. Thus, the amnestic effects of pretraining injections of Rompun are due to induction of state-dependent retention (SDR). Experiment 2 was designed to determine whether SDR would occur with alternative drugto-training intervals. Nine possible intervals were sampled, ranging from drug administration $90 \mathrm{sec}$ pretraining to $30 \mathrm{~min}$ posttraining. No evidence of retrograde effects were obtained: under the conditions employed, the drug must be administered at least $60 \mathrm{sec}$ prior to training for memory impairment to occur.
\end{abstract}

Drug-induced state-dependent retention (SDR) is a wellestablished phenomenon in which an animal is trained while some particular chemical condition (drug state) prevails, and when the animal is subsequently tested under drug conditions different from that present during learning, a response decrement is observed. Several of the drugs found to have SDR properties are classified as sedative and analgesic drugs. Classic sedative drugs that have been shown to produce SDR include alcohol, barbiturates, and benzodiazepines (Overton, 1966). The SDR-producing sedative drugs appear to possess a common biological mechanism: their sedative action has been suggested to be one of facilitating the activity of GABA, a major inhibitory neurotransmitter in the CNS (Grilly, 1989; Hamilton \& Timmons, 1990). In the present study, we examined whether xylazine (Rompun), a sedative/analgesic drug that is not believed to operate through GABA agonistic mechanisms, could produce SDR.

Use of xylazine in animal surgical procedures has become increasingly popular in recent years; therefore, the drug's effects on learning and memory may well be of importance to investigators. When injected alone or concurrently with anesthetics, xylazine induces sedation, analgesia, and muscle relaxation through its actions on the CNS. Numerous studies have shown that xylazineinduced CNS depression occurs through the drug's highly specific agonist effects on alpha-2 adrenergic receptors. Hsu (1981) showed that the effects of xylazine were prevented by alpha- 2 adrenergic-blocking agents (e.g., yo-

The research reported in this paper was supported in part by NIMH Grant 37535 to D.C.R. Correspondence should be addressed to D. C. Riccio, Department of Psychology, Kent State University, Kent, $\mathrm{OH}$ 44242 . himbine, tolazine, piperoxan, and phentolamine), but were not antagonized by other neurotransmitter-blocking agents, including alpha-1 adrenergic-blocking agents.

\section{EXPERIMENT 1}

\section{Method}

Subjects. Thirty-five adult (350-490 g) male Sprague-Dawleyderived rats served as subjects. The subjects were housed individually in hanging wire-mesh cages in a colony maintained on a 15:9-h light:dark cycle. All experimentation was carried out during the middle $5 \mathrm{~h}$ of the light cycle. Food and water were available ad lib in the home cage throughout the experiment. Two days prior to use, the subjects were handled for $5 \mathrm{~min}$ each day and randomly assigned to groups. Groups contained 9 subjects each, except for a single group with 8 subjects (due to subject mortality).

Apparatus and Procedure. Experiment 1 used a fear-conditioning (passive-avoidance) paradigm to determine whether xylazine produces SDR effects in an anterograde procedure. That is, the drug was administered prior to passive-avoidance training. The training procedure involved one-trial learning in which rats were placed into the white (safe) compartment of a $48 \times 19 \times 24 \mathrm{~cm}$ black-and-white shuttlebox with a grid floor (2-mm grids spaced $1 \mathrm{~cm}$ apart), followed by lowering of a guillotine dividing door and administration of a 3-sec nonescapable shock $(150 \mathrm{~V})$ once the subject placed all four feet into the black side of the shuttlebox. The subjects were immediately removed from the apparatus and returned to their home cages after receiving the shock.

All injections were administered IP at a dose of $3 \mathrm{cc} / \mathrm{kg}$, precisely $50 \mathrm{sec}$ prior to the subject being placed into the shuttlebox. (Once the subject was placed into the apparatus, another $10 \mathrm{sec}$ passed before the partition was lifted.) The xylazine injection was a $30 \%$ drug/70\% $\mathrm{H} 2 \mathrm{O}$ solution. Group 1 (S/Tr/S) subjects received saline and training on Day 1 and were tested on Day 2 after a subsequent saline injection. Groups $2(R / T r / S)$ and $3(R / T r / R)$ received xylazine and training on Day 1; however, only Group 3 received a subsequent xylazine injection on Day 2 (Group 2 received saline on Day 2). Group $4(R / N o T r / R)$ also received xylazine on both days; however, they were allowed to cross over in the training task without receiving shock. Group 4 served as a control for the same 


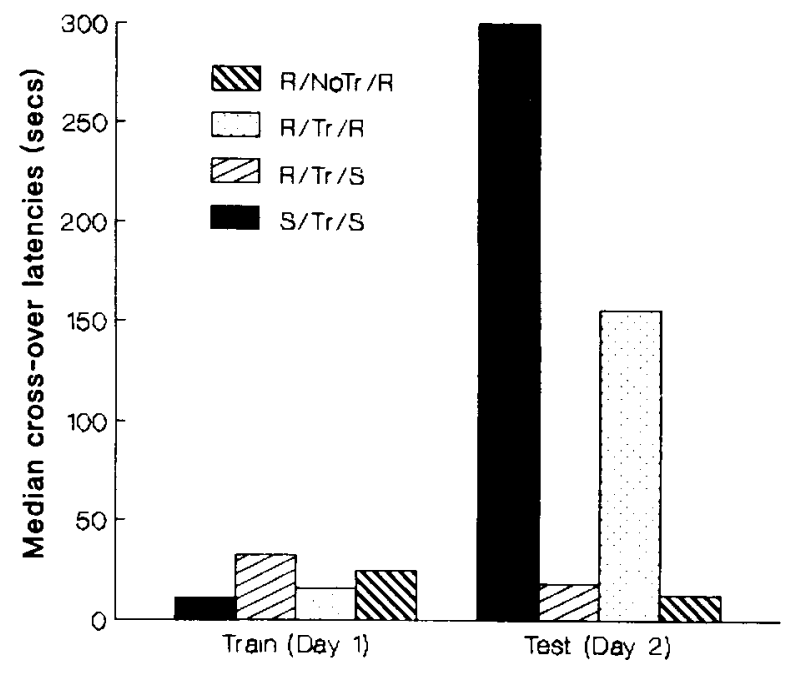

Figure 1. Median crossover latencies for training (Day 1) versus test (Day 2) trials. $R=$ Rompun, $S=$ saline, $T r=$ trained, NoTr $=$ not trained.

state condition, allowing us to evaluate whether any increase in test latency was due to buildup of the drug.

At testing, which occurred $24 \mathrm{~h}$ after the Day 1 training session, each rat received its appropriate injection $50 \mathrm{sec}$ before being placed into the white (safe) compartment of the shuttlebox. Latency to cross over to the black chamber, with a ceiling score of $300 \mathrm{sec}$, was recorded as the dependent variable.

\section{Results}

A Kruskal-Wallis analysis of variance (ANOVA) on the initial latency scores of Day 1 revealed no significant differences among the four experimental groups $[\mathrm{H}(3)=$ $7.10, p<.05]$. That is, the crossover latencies for all four groups did not differ prior to their receiving the training shock. A Kruskal-Wallis ANOVA on latency scores from Day 2 revealed a significant overall treatment effect $[H(3)=12.13, p<.01]$. Subsequent Mann-Whitney pairwise comparisons revealed significantly longer test latencies for the matched groups $(\mathrm{S} / \mathrm{Tr} / \mathrm{S}$ and $\mathrm{R} / \mathrm{Tr} / \mathrm{R})$ as compared to the mismatched, $R / T r / S$, group [S/TR/S vs. $\mathrm{R} / \mathrm{Tr} / \mathrm{S}, U=11.5, p<.01 ; \mathrm{R} / \mathrm{Tr} / \mathrm{R}$ vs. $\mathrm{R} / \mathrm{Tr} / \mathrm{S}, U=$ 8.0, $p<.01]$. Mann-Whitney comparisons also revealed a significant difference between Groups $R / T r / R$ and $\mathrm{R} / \mathrm{NoTr} / \mathrm{R}[U=11.0, p<.01]$, indicating that the long latencies of the $R / T r / R$ group were not due to drug buildup. Also, Groups $S / T r / S$ and $R / T r / R$ did not differ significantly, and Groups R/Tr/S and R/NoTr/R did not differ significantly (see Figure 1).

\section{Discussion}

These data indicate that xylazine produces statedependent retention. Rats receiving the drug $60 \mathrm{sec}$ before training show poor performance when tested without the drug. This decrement is not attributable to sensory or associative impairment, as readministration of the drug at testing results in significantly improved performance. Since an untrained group receiving the same drug regimen does not show long latencies (Group R/NoTr/R), the performance of Group $R / T r / R$ (trained and tested with Rompun) reflects memory retrieval rather than motor impairment produced by drug exposure. Thus, Rompun, like several other sedative agents, appears to have dissociative properties.

\section{EXPERIMENT 2}

Experiment 1 provided evidence that Rompun has dissociative properties when administered $60 \mathrm{sec}$ before training, that is, an anterograde memory deficit. This 60 sec interval presumably allowed the drug to begin to take effect. However, since the drug state persisted after training, an alternative explanation could be that xylazine has a retrograde effect on memory. This latter explanation, a subtle but potentially important methodological concern applicable to many "anterograde" paradigms (Riccio, Richardson, \& Guanowsky, 1983), is highly plausible here considering that a 3-cc/ $\mathrm{kg}$ dose of Rompun visibly sedated a rat for 2-3 h. However, the SDR effects induced by the classic sedative drugs, such as barbiturates and benzodiazapines, have generally been shown to be limited to anterograde amnestic effects (Overton, 1978; but see Chute \& Wright, 1973). Thus, if the SDR-producing effects of Rompun are similar to those of the classic sedatives, we should not expect to observe effects on memory in a strictly retrograde paradigm. Experiment 2 was designed to examine whether SDR would occur with alternative drug/training intervals, including Rompun administration after training was completed.

\section{Method}

Subjects. Experiment 2 involved 36 adult $(360-550 \mathrm{~g})$ male Sprague-Dawley-derived rats maintained under the same conditions as those employed in Experiment 1.

Apparatus and Procedure. The same black-and-white shuttlebox was used as in Experiment 1. Because difficulty exists in determining the precise interval between drug injection and the onset of the drug "state," Experiment 2 used a design in which groups received Rompun injections ranging from $90 \mathrm{sec}$ pretraining to $30 \mathrm{~min}$ posttraining. These intervals are in contrast to those in $\mathrm{Ex}$ periment 1 , in which all injections were given $60 \mathrm{sec}$ before training or test. Thus, this experiment included nine groups (4 subjects per group), each of which was given an IP injection of Rompun on Day 1 and saline on Day 2. Each group received its injections at the appropriate point along a continuum of $15-\mathrm{sec}$ intervals beginning with the 90 -sec pretraining group ( +90 group) and continuing through the immediate posttraining group ( -0 group). The exception was the 30 -min posttraining (long delay) group ( -1800 group) (see Figure 2).

As in Experiment 1, testing occured $24 \mathrm{~h}$ after training. Before testing, each group received a saline injection at the same $\mathrm{drug} / \mathrm{ma}$ nipulation interval as they received the Rompun injection on Day 1. Latency to cross over to the black chamber, with a ceiling score of $300 \mathrm{sec}$, was again recorded as the dependent variable of interest.

\section{Results}

A Kruskal-Wallis ANOVA on the initial latency scores from Day 1 revealed no significant differences among the initial cross-over latencies for the nine groups $[\mathrm{H}(8)=$ 


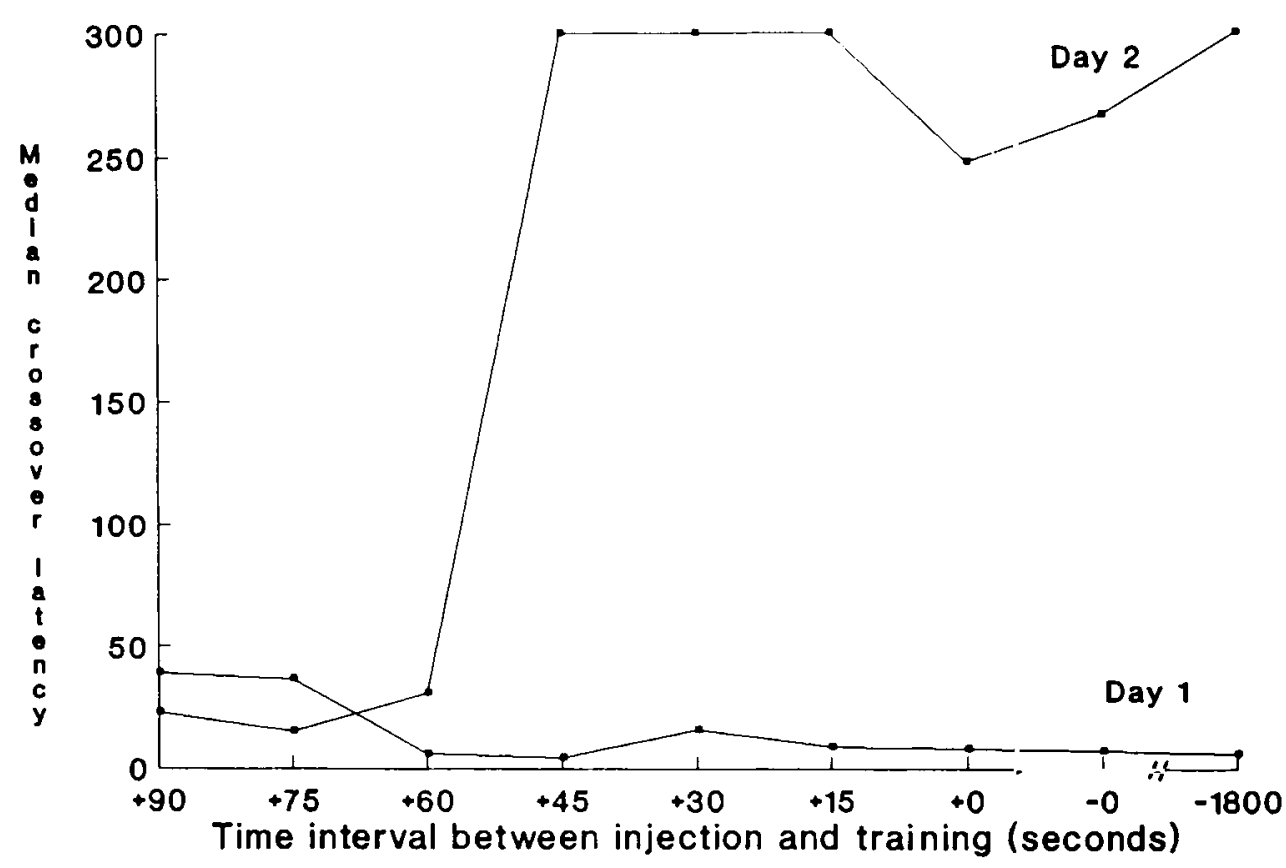

Figure 2. Median crossover latencies as a function of the time interval between injection and training (or vice versa). All groups were injected with Rompun on Day 1 and saline on Day 2. Groups left of the vertical bar $(+90$ through +0$)$ received both injections prior to training and test; those to the right $(-0$ and -1800$)$ received their Day 1 injections after training.

$12.11, p>.10]$. A Kruskal-Wallis test on latency scores from Day 2 revealed a significant overall treatment effect $[H(8)=14.57, p<.10]$. Subsequent Mann-Whitney pairwise comparisons indicated no significant differences between Groups $+45,+30,+15,+0,-0,-1800$. Also, Groups $+60,+75$, and +90 did not differ among each other. Therefore, given the small $n s /$ group but the clear functional relationship, a final Mann-Whitney comparison was performed on the collapsed Day 2 scores of Groups $+90,+75$, and +60 versus Groups $+45,+30$, $+15,+0,-0$, and -1800 . This final test indicated that the two collapsed groups differed significantly $(z=3.31$, $p<.001$ ) (see Figure 2).

\section{Discussion}

Experiment 2 indicated that the SDR capabilities of Rompun are very time specific. The results suggest that SDR only occurs when Rompun is injected at least $60 \mathrm{sec}$ before training. Any shortening of the drug/training interval, even by only $15 \mathrm{sec}$, completely abolishes the dissociative effects of the drug. Lengthening the drug-totraining interval beyond $60 \mathrm{sec}$ does not seem to cause a significant change in the dissociative effects; however, this could be a "floor effect" resulting from our dependent measure.

\section{GENERAL DISCUSSION}

The combined results of Experiments 1 and 2 suggest that Rompun does, indeed, produce SDR; however, the
SDR effects are limited to the conditions in which administration of the drug occurs at least $60 \mathrm{sec}$ prior to training. Thus, Rompun seems to produce SDR effects when used in an anterograde design (drug/training), but not when used in a retrograde design (training/drug). Although the -0 and -1800 conditions were the only groups that actually received the Rompun injection after training, the $+0,+15,+30$, and +45 groups can arguably be considered retrograde conditions because of the delayed onset of the drug. Collapsed together, these six groups provide substantial evidence against a retrograde effect. This asymmetrical finding is not suprising at all since classic SDR drugs such as pentobarbital are also presumed to work in the same limited fashion.

In conclusion, we have shown that Rompun does produce SDR effects even though its CNS actions probably do not involve a GABA agonist role, as do the traditional sedative/analgesic SDR drugs. Rompun is a very specific agonist at alpha-2 adrenergic receptors (autoreceptors), and so its SDR effects would seem to be mediated through this role. Although the biochemical effects of Rompun do not seem to coincide with the traditional SDR drugs, its behavioral effects may well be expected to generalize to these drugs. A study by Colpaert and Janssen (1985) showed that clonidine, another alpha-2 agonist, produced generalization with Rompun on a drug-discrimination procedure. A previous study by D'Mello (1982) indicated that clonidine generalized to the barbiturate amylobarbitone and the benzodiazepine chlordiazepoxide in a similar drug-discrimination procedure. Although not directly 
related, these studies suggest that Rompun should generalize behaviorally to the traditional SDR drugs of the barbiturate and benzodiazepine classes.

\section{REFERENCES}

Chute, D. L., W WIGHT, D. C. (1973). Retrograde state-dependent learning. Science, 180, 878-879.

Colpaert, F. C., \& Janssen, P. A. J. (1985). Discriminative stimulus properties of xylazine in rat: Discriminability and effects of putative alpha-2 adrenoreceptor agonists and antagonists. Journal of Pharmacology \& Experimental Therapeutics, 235, 521-527.

D'MELLO, G. D. (1982). Comparison of the discriminative stimulus properties of clonidine and amphetamine in rats. Neuropharmacology, 21, 763-769.

Grully, D. M. (1989). Drugs and human behavior. Needham, MA: Allyn \& Bacon.
Hamilton L. W., \& Timmons C. R. (1990). Principles of behavioral pharmacology. Englewood Cliffs, NJ: Prentice-Hall.

Hsu, W. H. (1981). Xylazine-induced depression and its antagonism by alpha adrenergic blocking agents. Joumal of Pharmacology \& Experimental Theraputics, 218, 188-192.

OVERTON, D. A. (1966). State-dependent learning produced by depressant and atropine-like drugs. Psychopharmacologia, 10, 6-31.

Overton, D. A. (1978). Major theories of state dependent learning. In B. T. Ho, D. W. Richards III, \& D. L.Chute (Eds.), Drug discrimination and state dependent learning (pp. 283-318). New York: Academic Press.

Riccio, D. C., Richardson, R., \& Guanowsky, V. (1983). Is anterograde amnesia a special case of retrograde amnesia? Behavioral Neuroscience, 97, 512-515.

(Manuscript received November 14, 1991; revision accepted for publication February 6, 1992.) 